\title{
Le darwinisme méthodologique de Veblen
}

Jean-Jacques Gislain

\section{(2) OpenEdition \\ Journals}

Édition électronique

URL : http://journals.openedition.org/ei/440

DOI : $10.4000 /$ ei. 440

ISSN : 2553-1891

\section{Éditeur}

Association Économie et Institutions

\section{Référence électronique}

Jean-Jacques Gislain, "Le darwinisme méthodologique de Veblen », Économie et institutions [En ligne], 17 | 2011, mis en ligne le 31 janvier 2013, consulté le 20 avril 2019. URL : http:// journals.openedition.org/ei/440 ; DOI : 10.4000/ei.440

Ce document a été généré automatiquement le 20 avril 2019

Revue Économie et institutions 


\title{
Le darwinisme méthodologique de Veblen
}

\author{
Jean-Jacques Gislain
}

L'auteur remercie les rapporteurs pour leurs remarques pertinentes et demeure responsable des erreurs et omissions qui persisteraient.

1 Parmi les approches économiques inspirées par un modèle biologique, la conception évolutionnaire de Thorstein Veblen (1857-1929) a eu une influence fondatrice sur l'institutionnalisme. Les continuateurs et commentateurs de Veblen se sont donc interrogés sur la place et le rôle exacts du modèle biologique darwinien dans l'œuvre veblenienne. Les nombreuses références à Darwin et l'usage de la terminologie darwinienne par Veblen ont ainsi donné lieu à de nombreux commentaires et controverses. Ceux-ci ont essentiellement porté sur la question du «darwinisme» de Veblen en comparaison avec la théorie de Darwin ${ }^{2}$. Notre objet dans cet article n'est pas de prolonger l'étude de cette question posée en ces termes ${ }^{3}$. Nous déplacerons plutôt l'étude vers la question de la caractérisation du «darwinisme méthodologique » de Veblen tel qu'il le met effectivement en œuvre dans ses constructions théoriques. Ce ne sont donc pas tant les énoncés de principes méthodologiques formulés par Veblen, invoquant l'autorité scientifique de Darwin, qui retiendront notre attention que les concepts et applications théoriques proposés par Veblen lui-même, sans nécessairement se référer explicitement à Darwin ou utiliser sa terminologie.

2 Le «darwinisme méthodologique $»^{4}$ consiste à adopter une approche évolutionnaire conforme à celle de Darwin en biologie mais sans nécessairement adopter les hypothèses théoriques darwiniennes, notamment concernant les processus de variation, de sélection et de transmission des caractères. Le darwinisme méthodologique n'est donc pas réductible au seul darwinisme de Darwin ${ }^{5}$, même s'il a été le premier à le formuler. Comme pour le physicalisme méthodologique qui peut admettre diverses hypothèses sur la nature de la «matière » et du "mécanisme», sur les « états » et «mouvements » de la nature physique et leurs tendances à l'« équilibre» ou au "chaos», etc., comme pour l'individualisme méthodologique qui permet des hypothèses variées sur la nature des 
« individus », sur les formes de "rationalité » et de « délibération », sur les mécanismes d'«agrégation" des comportements, etc., il est possible aussi au darwinisme méthodologique de supporter plusieurs sortes d'hypothèses sur la définition de l'« espèce », sur les processus de "variation ", de « sélection », de "transmission » des caractères, etc., et ceci en rapport avec l'objet d'étude retenu. Le darwinisme méthodologique comme méthode de connaissance de la réalité évolutive ne se cantonne donc pas nécessairement au strict domaine de la biologie ou à la stricte mise en application mécanique des hypothèses de travail et concepts darwiniens à d'autres domaines de connaissance. En somme, le darwinisme méthodologique n'est pas réductible au seul darwinisme et à son application mécanique dans les domaines autres que la biologie. À cet égard, la philosophie pragmatiste de C. S. Peirce et John Dewey, qui aura une forte influence sur l'institutionnalisme, est un exemple typique, contemporain de Veblen, d'application du darwinisme méthodologique dans un domaine autre que la biologie et dont les hypothèses de travail s'écartent significativement de celles du strict darwinisme. A contrario, le darwinisme social, très influent aux États-Unis au tournant du siècle (Hofstadter 1944, Bannister 1979), relève d'une application purement mécanique de la théorie darwinienne et de ses hypothèses au domaine des faits de société.

3 L'une des grandes originalités de Veblen est d'avoir proposé une approche évolutionnaire fondée sur l'application du darwinisme méthodologique à l'objet d'étude spécifique qu'est l'activité économique, et sans reprendre intégralement les hypothèses théoriques darwiniennes et bien que la terminologie veblenienne puisse prêter à confusion sur ce point. C'est ce darwinisme méthodologique veblenien, dont certes les degrés de perfection et de cohérence sont discutables, qui retiendra donc notre attention. Veblen adhère au darwinisme méthodologique comme alternative, elle-même évolutionnaire, aux autres méthodes de connaissance économique et à partir de l'évaluation critique qu'il opère de ces dernières et de leurs résultats. Le contenu de cette critique veblenienne $(1898,1919 a, 1934)$ est assez bien connu et nous n'y reviendrons pas ici (Gislain \& Steiner 1995, chap. I et V). L'adoption du darwinisme méthodologique conduit alors Veblen (1898, $1906,1908,1925)$ à devoir répondre à quatre questions méthodologiques centrales pour appuyer la pertinence de l'approche évolutionnaire en économie. La première question concerne ce qu'il faut entendre par «évolution » (\$1), la seconde l'identification de la catégorie «espèce " pertinente en économie (\$2), la troisième le mode opératoire au travail dans le processus de « sélection naturelle », c'est-à-dire celui des « variations » des " caractères » et de leur processus de "sélection », le tout constituant une « dérivation " (variation-sélection) (§3). Enfin, la dernière question concerne le processus de «transmission» des nouveaux caractères sélectionnés, perpétuant ainsi l'espèce transformée (\$4). Les réponses que propose Veblen à ces quatre questions expliquent pour une bonne part l'architecture générale de sa généalogie des institutions économiques (\$5).

\section{L'évolution comme processus}

4 Que faut-il entendre par "évolution» dans le domaine des faits économiques? L'évolutionnisme dans les sciences sociales à la fin du XIX siècle répond que l'évolution est un progrès qui tend naturellement à améliorer les conditions de vie, les institutions et la richesse dans la société. Cette conception téléologique d'une loi de progrès qui soustendrait l'évolution n'est pas retenue par Veblen (Gislain 1999). Pour ce dernier, 
l'évolution ne doit pas être appréhendée avec un biais normatif'. L'évolution est de fait un processus de transformation de la réalité où antécédents et conséquents ne sont pas évaluables en termes de progrès mais uniquement analysables en termes généalogiques. À chaque moment du processus évolutif, une forme générée comme nouvel élément de l'activité économique succède à une autre dans le complexe contemporain de la vie économique. Dans cette optique fidèle au darwinisme méthodologique, l'évolution est donc appréhendée uniquement comme une succession de transformations de la réalité ; elle est un processus séquentiel non finalisé dont l'intelligibilité n'est pas en dehors des forces et conflits à l'œuvre dans le processus même.

5 L'approche évolutionnaire à laquelle adhère Veblen se donne donc pour projet la construction d'un savoir économique qui se déploie en un corpus théorique unifié et dont le noyau est « une théorie d'un processus, d'un développement séquentiel » $(1898,58)$. La théorie économique se doit alors d'être "une théorie scientifique [qui] est une formulation des lois des phénomènes en termes des forces efficientes au travail dans la séquence des phénomènes" (1901, 302). L'un des éléments centraux du darwinisme méthodologique, ici repris par Veblen, est ainsi de considérer que la réalité est sans cesse en évolution selon des processus causaux, cumulatifs, irréversibles, aveugles et opaques à la raison suffisante de l'observateur. Les processus de transformation évolutive de la réalité économique sont des enchaînements cumulatifs de causes et d'effets. Ils sont irréversibles car chaque nouvelle séquence de la chaîne causale est un fait nouveau non réductible à ses antécédents. Ils sont aveugles car sans finalité préétablie. De fait, ils sont donc opaques à une rationalisation déterministe et téléologique de la part d'un observateur simplement muni introspectivement de sa raison suffisante. En somme, ces processus, objets centraux de l'approche évolutionnaire, sont endogènes à la chose évolutive, ils sont matter of fact, c'est-à-dire objectivement autonomes du regard de l'analyste et donc indépendants cognitivement des préconceptions de ce dernier sur l'ordre du monde. Dans ces conditions, l'approche évolutionnaire requiert une posture de connaissance impersonnelle, totalement épurée de présupposés sur le sens et l'évolution des faits. Telle est la posture de connaissance du darwinisme méthodologique adoptée par Veblen : les faits économiques et en général les faits réels évolutifs ne sont pas des objets d'étude qui se réfléchissent naturellement dans la seule raison suffisante de l'analyste nourrie de ses préconceptions normatives. Ces objets d'étude sont soumis à des processus dont il faut prendre connaissance, certes rationnellement et par l'abstraction conceptuelle, mais selon la logique objective de ces processus. Une fois définis ce qu'il faut entendre par évolution et comment il faut la comprendre comme processus, l'étape méthodologique suivante consiste à définir le concept d"espèce' pertinent pour l'approche économique évolutionnaire.

\section{L'espèce institution économique}

6 Pour Veblen, la vie économique se présente comme un ensemble de faits soumis à des processus de sélection. Cet ensemble va des caractères de la nature humaine jusqu'aux structures économiques concrètes. Il recouvre donc tout l'éventail des composants et déterminants économiques, des habitudes mentales jusqu'aux individus physiques et leurs conditions matérielles d'existence. Différents groupes de faits dans cet ensemble peuvent être étudiés comme des " espèces ", tour à tour objet et agent de sélection selon un processus cumulatif d'interaction. Suivant ses besoins spécifiques, l'étude 
évolutionnaire s'attache alors à l'analyse de certains éléments de ce processus, en particulier les objets et agents de la sélection de l'espèce économique concernée par l'étude. Le passage suivant est particulièrement exemplaire de la complexité de la conception veblenienne concernant la multiplicité des « espèces " pertinentes à étudier, de la combinatoire et de l'enchevêtrement des processus interactifs de sélection:

«La vie de l'homme en société, tout comme celle des autres espèces, est une lutte pour l'existence, et donc un processus d'adaptation sélective. L'évolution de la structure sociale a été un processus de sélection naturelle des institutions. Les institutions humaines ont fait et font encore des progrès qui se réduisent en gros à une sélection naturelle des habitudes mentales les plus recevables et à un processus d'adaptation forcée des individus à leur milieu qui a changé au fur et à mesure que la société se développait, et qui changeait aussi les institutions sous lesquelles les hommes ont vécu. Les institutions elles-mêmes ne sont pas seulement les résultats d'un processus sélectif et adaptatif, qui façonne les types prédominants d'attitude et d'aptitude spirituelle; elles sont en même temps des méthodes particulières de vie et de relations humaines, et à ce titre elles sont à leur tour de puissants facteurs de sélection. etc... » $(1899,124)$

7 Veblen étudie nombreux de ces processus interactifs de sélection. Cependant, ce sont les processus de sélection de l'espèce « institution » économique qui retiennent le plus son attention et auxquels il donne la place centrale dans son analyse ${ }^{7}$. Ce choix donnera son nom à l'école de pensée économique dont il est considéré comme l'un des principaux initiateurs: l'institutionnalisme; et même si ce n'est qu'en 1918 que W. H. Hamilton caractérisera ce qu'est l'économie « institutionnelle» (Hamilton 1919; Adair \& Gislain 2012). Veblen définit les institutions comme « des habitudes mentales prédominantes, des façons très répandues de penser les rapports particuliers et les fonctions particulières de l'individu et de la société » $(1899,125)$, de telle sorte que «l'adaptation des habitudes mentales, c'est le développement même des institutions » $(1899,140)$. Dès lors, le noyau dur de l'économie évolutionnaire est «la théorie d'une séquence cumulative d'institutions économiques formulée en termes de son propre processus » $(1898,77)$.

8 La définition des institutions proposée par Veblen se réfère à deux dimensions des habitudes mentales, celle relative au comportement individuel et celle relative au comportement social. Au niveau de la problématisation théorique, la première concerne la théorie de l'action, la seconde la théorie de l'institution. L'institution, de par cette double dimension, n'est donc pas une « interface » entre l'« individu » et la "société ». Elle est à la fois constitutive de l'acteur individuel comme être pensant et agissant - elle est une «habitude mentale » qui guide l'action individuelle -, et constitutive d'un fait social objectif - elle participe d'un "système conventionnel de vie " qui s'impose objectivement aux acteurs individuels sous formes de règles de conduite prescrites, socialement et cognitivement admises et constitutives d'un mode de vie ou théorie prédominante de la vie. L'institution est donc une « espèce » éminemment abstraite dont le concept ne s'épuise pas dans un référent identifiable dans la réalité matérielle, comme c'est le cas pour les espèces auxquelles s'intéresse Darwin. L'espèce institution est autant, sinon plus, une "force morale» (au sens de Durkheim) qu'un fait matériel. De plus, l'institution ne se réduit ni à une description empirique, comme le voudrait l'hyperréalisme de l'historicisme avec ses constructions typologiques d'acteurs et de faits sociaux, ni à une figure logique formelle, comme le voudrait le physicalisme de l'économie orthodoxe avec sa mécanique rationnelle des réactions individuelles. L'institution est une catégorie abstraite appartenant à une théorie de l'activité individuelle et à une théorie de l'institution comme modèle comportemental socialement 
déterminé. L'institution est un concept qui intègre donc à la fois la dimension individuelle de la cognition qui guide l'action, les habitudes mentales et leur évolution, et la dimension collective de l'action socialement contrôlée, les institutions s'imposant comme modes de vie et de pensée admis. L'institution est ainsi cette « espèce " bien particulière, un «matériel humain » comme dit Veblen, qui évolue selon une sorte de dialectique à deux pôles, parfois en phase ou décalés, parfois contradictoires ou se renforçant. Ces deux pôles sont l'action individuelle singulièrement vivante - l'évolution cognitive des habitudes mentales/institutions régissant l'action -, et l'action socialement admise - les institutions en tant que systèmes de vie et formes de connaissance s'imposant comme modes de pensée et d'action canoniques.

Le projet intellectuel de Veblen est donc très ambitieux. Il s'agit ni plus ni moins de tenter de résoudre l'un des problèmes centraux de la science sociale : rendre intelligible la vie et l'évolution de la société sans tomber dans les travers du constructivisme atomistique et physicaliste de l'individualisme méthodologique ou dans ceux du déterminisme totalisant et organiciste du holisme. Il s'agit, en d'autres termes, de concilier des théories de l'action et de l'institution qui conservent le caractère vivant-créateur de la pensée et de l'action individuelles... pourtant contrôlées par les institutions qui régissent les modes de penser et d'agir comme des forces morales et objectives dépassant l'individualité subjective des acteurs. Ce projet institutionnaliste initié par Veblen possède ainsi avec ce concept d'institution un potentiel analytique indéniable. Mais l'essentiel de la construction institutionnaliste reste à élaborer pour que la mise en œuvre du concept d'institution donne des résultats analogues à ceux de Darwin avec son concept d'espèce.

\section{Les dérivations : variation et sélection des caractères}

10 La troisième question méthodologique est alors cruciale car elle concerne l'identification du mode opératoire au travail dans le processus de «sélection naturelle » de l'espèce institution économique. Il s'agit plus précisément d'expliquer les «variations» des « caractères » et le processus de « sélection » de certains de ceux-ci pour la perpétuation de l'espèce. En somme, il s'agit de rendre compte du processus complet de "dérivation » (variation-sélection) à l'œuvre dans la transformation-évolution de l'espèce institution économique.

11 En conformité avec le darwinisme méthodologique et la terminologie établie par Darwin, Veblen défend l'idée d'une «sélection naturelle» des institutions économiques. Il reprend ainsi, concernant le second processus constitutif de la " dérivation ", l'hypothèse darwinienne selon laquelle les nouveaux «individus » de l'espèce qui survivent, et qui donnent donc sa nouvelle forme évolutive à l'espèce, sont ceux qui sont les plus aptes à perpétuer l'espèce dans les nouvelles conditions de son milieu de vie. En termes d'économie évolutionnaire, ce sont les nouvelles institutions économiques les plus aptes à perpétuer, dans les nouvelles conditions de vie matérielle, l'espèce institution dont elles sont issues qui survivent et impriment donc leurs formes évolutives à leur espèce institutionnelle. Ainsi, parmi les nouvelles formes potentielles d'activité économique, seules celles s'inscrivant dans la phylogenèse de l'institution dominante peuvent être candidates et donc admissibles au maintien du complexe d'institutions dérivées de cette institution dominante. Pour qu'une espèce institutionnelle se perpétue, il faut donc que 
son phylum soit respecté. Voilà pour le processus de "sélection" lui-même admissibilité (phylogenèse) et meilleur aptitude (compétition parmi les plus aptes) - qui est très darwinien chez Veblen.

12 En effet, pour Veblen, chacune des institutions économiques "dérivées" a été « sélectionnée » pour son aptitude, relativement au milieu historique du complexe des institutions dans lequel elle s'inscrit et selon l'évolution des conditions matérielles d'existence, à perpétuer l'institution primaire de la propriété, soit directement en transformant les formes de la propriété (1904, 1919b, 1923), soit indirectement en transformant l'une de ses institutions secondaires, la consommation et le loisir (1899). Dès lors, pour comprendre pourquoi une forme institutionnelle a été sélectionnée, il est nécessaire de la replacer dans son complexe institutionnel ou, comme dit Veblen, dans un système conventionnel de vie, c'est à dire un agrégat d'institutions dominantes à un moment donné de l'évolution sociale et qui prend la forme spirituelle d'une théorie prédominante de la vie $(1899,125-126)$. Ce système de vie est caractérisé par une institution primaire, en l'occurrence la propriété/mentalité propriétaire, à la source d'un phylum, d'une série évolutive d'institutions dérivées. Les institutions secondaires " sélectionnées » viennent s'agréger au complexe du système de vie et sont dérivées de l'institution primaire par un processus de sélection retenant les variations institutionnelles adaptées aux modifications de l'environnement économique. Sont donc sélectionnées les seules institutions qui sont aptes à faire survire l'institution primaires dont elles dérivent. En somme, pour Veblen, dans une société dominée par l'institution primaire de la propriété, les processus de sélection « se réduisent en gros à une sélection naturelle des habitudes mentales les plus recevables » $(1899,124)$, c'est-à-dire les plus conformes à la survie de l'espèce institutionnelle en évolution, celles issues de la propriété. Les transformations de la réalité institutionnelle confirment sans cesse la réalité antérieure et son principe constitutif : la mentalité propriétaire. Cette survie de la réalité institutionnelle apparaît alors, à tout moment de l'évolution, comme "naturelle " aux yeux des acteurs. Elle renforce ainsi les habitudes mentales dominantes issues de la mentalité propriétaire et contribue à perpétuer la «sélection naturelle» des nouvelles institutions « recevables ».

13 Ainsi dans la représentation que suggère Veblen de l'évolution économique, la phylogenèse des institutions économiques a toutes les apparences, dans ses résultats, d'un arbre généalogique de type darwinien. Les formes successives de l'espèce ont toutes été sélectionnées selon un même phylum et selon un enchainement cumulatif qui fait de chacune, à un certain degré de ramification continue, une transformation de l'espèce originelle. Mais là s'arrête l'adhésion de Veblen au darwinisme canonique. En effet, Veblen se sépare significativement du darwinisme, bien qu'il utilise une terminologie darwinienne, quant aux hypothèses théoriques sur le processus de "variation » des caractères. Il n'adhère pas à la théorie darwinienne de la variation aléatoire des nouveaux caractères à l'origine des possibilités de transformation de l'espèce. Pour Veblen, la variation des nouveaux caractères n'est pas de type aléatoire - un fait exogène inexpliqué, une entéléchie - mais le fait d'un processus double : l'un de création humaine, l'autre de causalité généalogique, une conséquence générée par des antécédents identifiables dans la phylogenèse institutionnelle.

14 Le premier processus à l'origine de l'apparition d'un nouveau caractère, éventuellement sélectionnable comme variation effective, résulte, selon Veblen, d'un des éléments constitutifs de la nature humaine : l'instinct de la curiosité gratuite (idle curiosity), c'est-à- 
dire «la curiosité qui pousse les hommes vers les inventions ingénieuses et déconcertantes " $(1899,100)$. Cet instinct alimente en créations et nouveautés les mentalités et comportements individuels et génère, de la sorte, une palette de possibles nouveaux caractères potentiellement constitutifs de variations. Cependant, et c'est le second processus, seuls sont retenus à la candidature pour la sélection les nouveaux caractères qui peuvent s'inscrire dans la continuité de l'espèce, dans la phylogenèse des séquences cumulatives et évolutives de l'espèce elle-même, et qui sont aussi les conséquences issues de la logique interne évolutionnaire à l'œuvre dans le système institutionnel lui-même. Le premier aspect de ce second processus est déjà connu. Il s'agit de l'admissibilité des nouveaux caractères à la sélection. En revanche, le second aspect, le fait que les nouveaux caractères admissibles sont les conséquences des institutions ellesmêmes, est une particularité de l'utilisation du darwinisme méthodologique par Veblen.

Veblen soutient que les seules variations institutionnelles possibles sont les produits d'un processus endogène causal et cumulatif au travail dans l'efficience même des institutions à s'adapter aux conditions environnementales changeantes. En d'autres termes, Veblen soutient que les institutions d'une espèce institutionnelle génèrent elles-mêmes les conditions objectives (les caractères candidats à la variation sélectionnée) de leur adaptation future. Le cas que propose Veblen à cet égard est paradigmatique. En effet, Veblen souligne, à propos de la question du moment d'apparition historique de la classe de loisir : «Quand bien même la classe de loisir ne se serait pas instituée en même temps que la propriété individuelle, par l'effet du déshonneur qui dès lors s'attachait aux occupations productives, elle aurait pris naissance comme l'une des conséquences de la propriété » (1899, 28-29, souligné par nous). Il y a donc bien un lien de causalité, une relation de cause à effet entre un antécédent, l'institution de la propriété, et un conséquent, une "variation » institutionnelle : l'apparition de la classe de loisir avec ses caractères institutionnels que sont les institutions du loisir et de la consommation «sélectionnées " pour leurs aptitudes à faire survivre et se développer la mentalité propriétaire dont elles sont issues. De plus, Veblen cherche à qualifier le processus de causalité à l'œuvre entre l'antécédent et le conséquent. Dans le cas de l'institution du loisir comme conséquence de l'institution de la propriété, Veblen précise que le processus de causalité en est un de dérivation, de secondarité et d'inversion mentale :

Cette valeur immédiate et subjective du loisir et des autres preuves de richesse est en grande partie secondaire et dérivée. Si elle reflète l'utilité du loisir, ce moyen de s'attirer le respect d'autrui, elle résulte aussi d'une substitution mentale. Il était admis qu'en accomplissant un travail, on faisait par convention l'aveu de l'infériorité de ses forces ; un court-circuit de l'esprit amène à considérer le travail lui-même comme intrinsèquement vil $(1899,28$, souligné par nous)

16 Ainsi, par delà l'analyse généalogique qui cherche à décrire la phylogenèse d'une institution secondaire à partir d'une institution primaire, Veblen propose de procéder à la reconstruction logique de cette phylogenèse, c'est-à-dire de rendre intelligible le processus causal cumulatif aveugle et opaque qui produit la dérivation (variation et sélection) d'une nouvelle institution. L'étude du processus de «variation » des caractères sélectionnés de l'espèce, qui est éludée dans le darwinisme sous la figure magique du fait aléatoire, devient alors centrale dans l'analyse veblenienne. Elle offre à cette dernière la possibilité de développer la double dimension de l'analyse progressive de la cause à l'effet, c'est-à-dire la variation, et régressive de l'effet à la cause, c'est-à-dire la sélection.

Le processus de l'enchaînement causal cumulatif de la dérivation (variation et sélection) peut aussi bien être analysé sur le mode de la reconstitution des séquences successives 
qui ont conduit à la transformation d'une institution première en une série d'institutions dérivées, que sur le mode de la reconstruction rétrospective de ce processus à partir d'une institution secondaire. Ainsi, par exemple, le surplus économique, issu de la capacité productive de l'instinct du travail efficient, accaparé par les propriétaires a pour conséquence que ceux-ci s'adonnent à la consommation de luxe et à l'oisiveté, ces deux activités étant ainsi les caractères à l'origine des variations que sont les institutions secondaires du loisir et de la consommation. Inversement, l'analyse rétrospective peut montrer que parmi les multiples effets de l'institution de la propriété, ceux qui sont sélectionnés sont ceux qui perpétuent le plus efficacement la mentalité propriétaire, en l'occurrence les activités honorables de la consommation et du loisir. L'institution ayant " pris sa forme achevée » $(1899,29)$, c'est-à-dire la forme institutionnelle contemporaine la plus évoluée, offre alors le plus grand potentiel pour l'analyse évolutionnaire car, en tant que genre le plus évoluée dans une espèce, elle cristallise toute les caractéristiques de la phylogenèse de l'espèce. Par exemple, l'institution de la classe de loisir adopte sa forme achevée au stade moderne de la culture pécuniaire et prend ainsi son «sens plénier» $(1899,29)$, en particulier dans les comportements contemporains de consommation et loisir ostentatoires. Pour sa part, l'institution de la propriété évoluant vers le stade contemporain de la suprématie du capital financier relève son caractère originel d'institution parasitaire et de prédation (1904, 1919b, 1923).

Dans les multiples facettes de son œuvre, Veblen procède toujours comme si, en partant d'un point quelconque de l'arbre généalogique des institutions économiques, il était possible historiquement et logiquement aussi bien de le remonter que de le redescendre. Bien que fondé sur les principes du darwinisme méthodologique appliqués à l'étude de l'évolution des institutions économiques, le cadre analytique veblenien n'est donc pas strictement assimilable au darwinisme, ni d'ailleurs au lamarckisme; il faudrait plutôt parler de «veblenisme» pour le caractériser. A cet égard, la dernière question méthodologique centrale à laquelle Veblen se confronte est aussi révélatrice.

\section{Transmission des caractères}

Les particularités du mode de transmission des caractères institutionnels sont liées au fait que l'espèce institution économique n'est pas fondamentalement une entité physique, même si certaines de ses formes objectives concrètes s'inscrivent dans la matérialité. Constituée d'habitudes mentales, elle appartient à la «matrice culturelle » de l'humanité et elle s'inscrit donc dans une durée propre qui n'est pas celle biologique des êtres humains qui en ont porteurs. La transmission de ces caractères ne peut donc être " héréditaire » au sens darwinien du terme, c'est-à-dire par la génération physique. Dans ces conditions, la question de la transmission des caractères économiques institutionnels se pose différemment que pour les espèces biologiques. Le problème de la transmission des caractères « acquis » ne se pose pas vraiment car l'éducation, l'imitation et toutes les formes sociales de transmission des savoir faire et des savoir penser sont là pour $\mathrm{y}$ pourvoir. Dans le domaine des institutions économiques modernes, les établissements d'enseignement se vouent à cette tâche dont Veblen (1918) raille le zèle à former, avec l'aide du financement des milieux d'affaires, des étudiants bien " adaptés » à la mentalité propriétaire dominante. Ainsi formés, ils contribuent activement à la perpétuation de l'institution de la propriété et de ses dérivés. Tant qu'une nouvelle institution économique ne s'est pas imposée comme plus «recevable», la transmission des 
caractères des institutions économiques en place est assurée par le renouvellement effectif et jugé efficient des pratiques et droits économiques qu'elles supportent.

Dans ces conditions, l'analyse veblenienne ne se centre pas sur la question de la transmission des caractères économiques institutionnels mais plutôt sur leur non transmission ainsi, qu'inversement, sur le retour atavique de certains caractères ancestraux. De ce point de vue, l'analyse veblenienne se déploie à deux niveaux. Celui, général, de la capacité de l'institution de la propriété à se perpétuer contre ce qui, pour Veblen, est son ennemi héréditaire : l'instinct du travail efficient dans son esprit premier d'utilité (1914). La transmission ou la non transmission des caractères des institutions issues de la propriété est alors analysée en référence à cette opposition fondamentale. Le second niveau d'analyse, qui découle du premier, concerne alors la compétition à laquelle se livre les institutions dérivées de la propriété dans leur tâche de sauvegarde de la pérennité de l'institution primaire de la propriété. Les institutions dérivées de la propriété qui s'imposent dans cette fonction, et sont donc sélectionnées comme telles, font alors disparaitre les autres ou s'y substituent plus ou moins totalement. Dans ces processus d'éviction et de substitution, certains caractères ataviques peuvent alors réapparaître comme les plus adaptés à de nouvelles conditions de perpétuation de l'institution de la propriété. En somme, de l'état des forces dans le combat auquel se livrent les mentalités " propriétaire » et « technique » résulte les possibilités respectives de survie des institutions cérémonielles de la propriété. Dans un monde économique dominé par la propriété mais fortement influencé par les compétences techniques de production, l'institution de la consommation s'impose à celle de l'oisiveté. Inversement, dans un monde économique de prédation financière, les institutions du loisir ostentatoire et l'atavisme de la rapine reviennent à l'honneur, etc.

21 Les conceptions vebleniennes de la dérivation (variation-sélection) et de la transmission des caractères conduisent ainsi à penser que le darwinisme méthodologique de Veblen, et généralement l'institutionnalisme dont il est l'initiateur, s'écarte significativement du darwinisme stricto sensu. Cet aspect de la pensée veblenienne apparaît tout à fait clairement si on reconstruit l'architecture du l'« arbre généalogique » des institutions qui ressort implicitement de l'ensemble de l'œuvre de Veblen.

\section{Généalogie des institutions}

La structure de l'arbre généalogique des institutions, élaborée au cours de l'oeuvre de Veblen, peut être présentée de la façon suivante. À l'origine du phylum de la série évolutive des institutions économiques se présente une institution primaire. Celle-ci est l'institution de la propriété qui correspond à l'habitude mentale valorisant socialement la détention et la captation du surplus économique, c'est la mentalité propriétaire. De cette institution primaire dérivent des institutions secondaires qui en sont les formes successives plus évoluées. Les institutions secondaires peuvent être des dérivations directes de l'institution primaire de la propriété; ce sont les formes historiques successives de droits de propriété, de l'esclavage jusqu'à la propriété intangible du capital financier. Les institutions secondaires peuvent aussi être des dérivations indirectes dont les liens et le processus de génération à partir de l'institution primaire de la propriété sont plus opaques que dans le premier cas. Ces institutions secondaires indirectes sont principalement le loisir et la consommation, l'ostentation du gaspillage de temps/non travail et de richesses non objectivement utiles/futilités. Elles véhiculent et renforcent les 
habitudes mentales quant à l'honorabilité de la propriété et fonctionnent comme indicateurs de classement social dans le jeu spéculaire de la comparaison provocante, en particulier sous la forme moderne de la rivalité pécuniaire. Ces institutions secondaires indirectes sont elles aussi évolutives et peuvent se substituer les unes aux autres lorsqu'elles sont en concurrence quant à leur rôle de renforcement de la mentalité propriétaire. Les institutions de la consommation et du loisir, et leurs formes dérivées, s'imposent respectivement plus ou moins fortement selon des phases d'évolution du complexe d'institutions dominé par la mentalité propriétaire. Les institutions secondaires peuvent aussi se transformer en prenant les formes d'institutions subsidiaires. Ces formes apparaissent lorsque la mise en œuvre de certains attributs des institutions secondaires sont délégués à des personnes autres que les propriétaires, la gestion et la direction technique des entreprises à des salariés dans le cas des institutions secondaires directes, l'oisiveté et la consommation à la femme du propriétaire ou autres " délégués » à cette tâche de gaspillage ostentatoire dans le cas des institutions secondaires indirectes. Enfin, outre certaines formes institutionnelles ataviques qui peuvent survivre ou réapparaitre selon leur adéquation contemporaine au renforcement de l'institution dominante, par exemple l'institution du sport comme mise en scène des attributs guerriers ancestraux de la mentalité propriétaire, certaines institutions secondaires indirectes peuvent évoluer en formes mixtes conjuguant les traits les plus manifestes de la mentalité propriétaire et de la subsidiarité. Le phénomène de la mode, avec ses formes de gaspillage ostentatoire extrême et de délégation est, pour Veblen, le cas exemplaire de cette forme la plus évoluée de l'institution primaire de la propriété, l'expression de sa forme plénière.

Quatre degrés de transformation sont ainsi repérés par Veblen dans la structure de l'arbre généalogique des institutions économiques issues du tronc central qu'est l'institution primaire de la propriété. Cette structure et sa combinatoire de possibles dérivations donnent à l'arbre d'évolution des institutions une complexité de ramifications et un ensemble de variétés étonnants (Gislain 2000). Plus le processus d'évolution est avancé et multiplie les branches rapportées et plus la variété des institutions collatérales est grande et, donc, plus la compréhension des relations génétiques qu'elles entretiennent entre-elles est opaque à l'observateur contemporain. La multitude et l'apparence des brindilles périphériques de l'arbre généalogique, c'est-à-dire l'ensemble des habitudes mentales/institutions à l'œuvre à un moment de l'évolution, masquent leurs dérivations premières à partir du tronc central et ses racines: la mentalité/ institution propriétaire. L'objet d'étude principal de l'institutionnalisme évolutionnaire de Veblen est alors de rendre intelligible la chaîne des variations génétiques qui aboutit aux institutions contemporaines, d'expliquer les processus à l'œuvre dans la ramification institutionnelle.

En somme, il est vain de rechercher chez Veblen le contenu du «darwinisme », même dans une version moderne et conjuguée avec des éléments de lamarckisme (Hodgson 1992, Tilman 1996). Il est plus fructueux de voir dans l'œuvre de Veblen une tentative originale, certes plus ou moins réussie ${ }^{8}$, d'appliquer le darwinisme méthodologique à un objet d'étude spécifique : l'évolution des institutions économiques. 


\section{BIBLIOGRAPHIE}

Abecassis P., N. Coutinet et J.-P. Domin (2008), « Logiques industrielles versus droits sociaux : une application au secteur de l'assurance maladie complémentaire », dans J.-P. Domin, M. Maric, S. Delabruyère et $\mathrm{C}$. Hédoin (éds), Au-delà des droits économiques et des droits politiques, les droits sociaux , Paris, L'Harmattan, pp. 153-166.

Abecassis P. et J.-P. Domin (2009), «L'évolution des conventions médicales à travers leur discours. Les maux de la politique économique de santé », dans T. Barnay et F. Legendre (éds), Emploi et politiques sociales. Tome 1 : défis et avenirs de la protection sociale., Paris, Éditions de L'Harmattan, pp. 71-84.

Arnould M.-L., S. Pichetti et M.-O. Rattier, (2007), « Les contrats les plus souscrits auprès des organismes complémentaires en santé », Études et résultats, (575).

Arnould M.-L. et G. Vidal, (2008), « Typologie des contrats les plus souscrits auprès de complémentaires santé en 2006 ", Études et résultats, (663).

Barbier J.-C. et B. Théret (2004), Le nouveau système français de protection sociale, Paris, La découverte.

Barbot J. (2002), Les malades en mouvements. La médecine et la science à l'épreuve du sida, Paris, Balland.

Batifoulier P., J.-P. Domin et M. Gadreau, (2007), « Chapitre introductif. Politique de santé : la grande transformation ", Économie appliquée, 60 (1), pp. 5-36.

Batifoulier P., J.-P. Domin et M. Gadreau, (2007), « La gouvernance de l'assurance maladie au risque d'un État social marchand », Économie appliquée, 60 (1), pp. 101-126.

Batifoulier P., J.-P. Domin et M. Gadreau, (2008), « Mutation du patient et construction d'un marché de la santé. L'expérience française », Revue française de socio-économie, (1), pp. 27-46.

Benamouzig D. et J. Besançon, (2005), « Administrer un monde incertain : les nouvelles bureaucraties techniques. Le cas des agences sanitaires en France », Sociologie du travail, 47 (5), pp. 301-322.

Boltanski L. et È. Chiapello (1999), Le nouvel esprit du capitalisme, Paris, Gallimard, nrf essai.

Boltanski L. et L. Thévenot (1991), De la justification. Les économies de la grandeur, Paris, Gallimard.

Callon M. (1998), "Introduction: the embeddedness of economic market in economics", dans M. Callon (éd), The laws of the market, Oxford, Blackwell Publishers, pp. 9-46.

Callon M., (2002), « Pour en finir avec les incertitudes? ", Sociologie du travail, 44 (2), pp. 261-267.

Callon M., C. Meadel et V. Rabeharisoa, (2001), « L'économie des qualités », Politix, 52 (1), pp. 211-239.

Callon M. et F. Muniesa, (2003), « Les marchés économiques comme dispositifs de calcul », Réseaux ,(122), pp. 189-233.

Chambaretaud S. et D. Lequet-Slama, (2003), « Les systèmes de santé danois, suédois et finlandais, décentralisation, réforme et accès aux soins », Études et résultats, (214). 
Chamberlin E.-H., (1953), “The product as an economic variable”, Quaterly journal of economics, 67 (1), pp. 1-29.

Cohu S., D. Lequet-Slama et P. Volovitch, (2005), « Réforme des systèmes de santé allemand, néerlandais et suisse et introduction de la concurrence », Études et résultats, (445).

Coriat B. et O. Weinstein, (2004), «Institutions, échanges et marchés », Revue d'économie industrielle, (107), pp. 37-62.

Coriat B. et O. Weinstein, (2005), « La construction sociale des marchés », La lettre de la régulation, (53).

Demange G. et P.-Y. Geoffard, (2004), « À quoi peut servir la concurrence en assurance santé ? ", Revue d'économie politique, 114 (5), pp. 595-609.

Domin J.-P. (2003), « Crise sociale et institutionnalisation des solidarités : une application au système de santé (1890-1940)", dans P. Guillaume (éd), Les solidarités (2). Du terroir à l'État., Bordeaux, Éditions de la MSH d'Aquitaine, pp. 73-93.

Domin J.-P., (2006), « Du droit à la santé au risque sanitaire : une rupture paradigmatique ? ", Économie appliquée, 59 (2), pp. 111-136.

Domin J.-P., (2006), « La démocratie sanitaire participe-t-elle à la construction d'un consommateur de soins ? ", Journal d'économie médicale, 24 (7-8 ), pp. 427-438.

Domin J.-P., (2003), « La santé entre marché et services publics », La Pensée, (333), pp. 5-18.

Domin J.-P. et A.-S. Ginon (2007), « L'instauration de mécanismes incitatifs en faveur des contrats d'assurance maladie complémentaire : quels effets sur les inégalités en santé », dans $P$. Batifoulier, A. Ghirardello, G. Larquier (de) et D. Rémillon (éds), Approches institutionnalistes des inégalités en économie sociale, Paris, Éditions de l'Harmattan, pp. 233-244.

Esping-Andersen G. (1999), Les trois mondes de l'État-providence. Essai sur le capitalisme moderne, Paris, Puf.

Ewald F. et D. Kessler, (2000), « Les noces du risques et de la politique », Le débat, (109), pp. 55-72.

Eymard-Duvernay F., (1989), « Convention de qualité et formes de coordination », Revue économique, 40 (2), pp. 329-359.

Fassin D. (1998), « Les politiques de la médicalisation », dans P. Aïach et D. Delanoë (éds), L'ère de la médicalisation. Ecce homo sanitas, Paris, Économica, pp. 1-13.

Fenina A., Y. Geffroy et M. Duée, (2008), « Les comptes nationaux de la santé en 2007 », Études et résultats, (655).

Foucault M. (2004), Naissance de la biopolitique. Cours au Collège de France.1978-1979, Paris, Gallimard, Éditions du Seuil.

Foucault M. (1963), Naissance de la clinique, Paris, Puf.

Francesconi C., M. Perronnin et T. Rochereau, (2006), « La complémentaire maladie d'entreprise : niveaux de garanties des contrats selon les catégories de salariés et le secteur d'activité », Questions d'économie de la santé, (112).

Gaudillière J.-P. et P.-B. Joly, (2006), « Appropriation et régulation des innovations biotechnologiques : pour une comparaison transatlantique », Sociologie du travail, 48 (3), pp. 330-349.

Hassenteufel P., (1997), «Le “plan Juppé” : fin ou renouveau d'une régulation paritaire de l'assurance maladie? ", Revue de l'IRES, (24), pp. 175-189. 
Jaunait A. (2005), Comment pense l'institution médicale? Une analyse des codes français de déontologie médicale, Paris, Dalloz.

Kerleau M., (1998), «L'hétérogénéité des pratiques médicales, enjeu des politiques de maîtrise des dépenses de santé », Sciences sociales et santé, 16 (4), pp. 5-32.

Marical F. et T. Saint-Pol (de), (2007), « La complémentaire santé : une généralisation qui n'efface pas les inégalités ", INSEE Première, (1142).

Moureau N. et D. Sagot-Duvauroux (2006), « La construction sociale d'un marché : le cas du marché des tirages photographiques ", dans F. Eymard-Duvernay (éd), L'économie des conventions. Méthodes et résulats, Paris, Éditions de la Découverte, pp. 45-60.

Pignarre P. (2004), Comment sauver (vraiment) la sécu, Paris, Éditions La Découverte.

Rabeharisoa V. et M. Callon (1999), Le pouvoir des malades, Paris, Les presses de l'école des Mines.

Razac O. (2006), La grande santé, Paris, Climats/Flammarion.

Steffen M., (1987), « Les médecins et l'État en France », Politiques et management public, 5 (3), pp. 19-39.

Steudler F., (1977), « Médecine libérale et conventionnement », Sociologie du travail, 19 (2), pp. 176-198.

Théret B., (2007), « Les transformations de l'État social français depuis 1975 : une analyse centrée sur les politiques de santé et menée au regard des standards typologiques et théoriques du changement institutionnel ", Économie appliquée, 60 (1), pp. 69-100.

Tordjman H., (2004), "How to study markets. An institutionalist point of view", Revue d'économie industrielle, (107), pp. 19-36.

Torny D., (1995), « Mon corps est-il une bagnole ? Socio-fiction assurantielle », Revue française des affaires sociales, 59 (4), pp. 67-78.

Tournay V., (2007), «Produire l'objectivité médicale : un système expérimental réplicatif de standardisation », Sociologie du travail, 49 (2), pp. 253-267

\section{NOTES}

2. Sur la question du « darwinisme » de Veblen, voir : A. L. Harris (1934), I. Murphree (1959), L. B. Jones (1986), H. H. Liebhafsky (1988), G. M. Hodgson (1992), M. Boyles \& R. Tilman (1993), A. Jennings \& W. Waller (1994, 1998), R. Tilman (1996a, 1996b), M. Rutherford (1998) et ces dernières années G. M. Hodgson (2001, 2003, 2004a, 2004b, 2008). Pour un condensé des critiques du « darwinisme » de Veblen, voir G. Liagouras (2009).

3. G. M. Hodgson (2002, 2008 ; Hodgson \& Knudsen 2006) et d'autres (Aldrich et al 2008, Hédoin 2010), en se fondant notamment sur l'œuvre séminale de Veblen, défendent ce qu'ils appellent le "darwinisme généralisé », c'est-à-dire le fait que tout processus d'évolution biologique ou socioculturel est régi par les trois principes darwiniens de variation, de réplication et de sélection. Cette thèse est contestée par les partisans de «l'hypothèse de continuité » (il n'y a pas d'identité ontologique entre les processus d'évolution biologique et socioculturel) selon lesquels Veblen n'aurait utilisé les notions darwiniennes que de façon métaphorique (Cordes 2006, 2007, 2009). Ce débat tourne encore autour de la stricte question du «darwinisme » de Veblen, ce qui n'est pas ici notre préoccupation en tant que telle.

4. N. Rescher (1977, p. 142) utilise le terme de « method darwinism », nous lui préférons celui de darwinisme méthodologique (methodological darwinism). 
5. Sur les débats actuels autour du « darwinisme » de Darwin, voir P. Tort (1996).

6. C'est ce qui différencie l'approche évolutionnaire de l'approche évolutionniste.

7. Lorsque Veblen (1913a, 1913b) s'aventure dans l'étude des différents caractères des "races » humaines et de leur actions respectives sur les processus évolutifs de l'activité économique, son « racialisme » (Tilman 1996, 57) est difficilement défendable.

8. En particulier, deux éléments de l'analyse veblenienne posent problème. Tout l'édifice de la construction généalogique de Veblen repose sur ses postulats discutables quant aux « instincts » constitutifs de la «nature humaine ». En second lieu, Veblen propose sa fameuse "dichotomie » entre les deux logiques s'affrontant dans l'évolution institutionnelle: celle pécuniaire et cérémonielle sous tendue par la rivalité provocante et au phylum des institutions "propriétaires"; et celle industrieuse et instrumentalement efficace sous tendue par l'instinct du travail efficient et au fondement de l'esprit «ingénieur» des travailleurs. Plus Veblen avancera dans son œuvre, plus il soutiendra la possibilité que la logique industrielle puisse l'emporter sur la logique financière, allant jusqu'à prôner le «soviet des ingénieurs » (Veblen1921). Or, sauf revirement historique improbable, toute l'analyse généalogique de l'évolution des institutions économiques proposée par Veblen, et que nous venons de présenter, laisse plutôt penser que la perpétuation et le renforcement des institutions propriétaires sont inéluctables, la logique industrieuse étant fatalement instrumentée par la rivalité pécuniaire, comme s'il y avait un ordre hiérarchique de subordination d'un terme de la dichotomie par l'autre.

\section{RÉSUMÉS}

Thorstein Veblen offre une approche de l'évolution des institutions économiques fondée sur le darwinisme méthodologique. Veblen doit alors répondre à quatre questions méthodologiques importantes. La première concerne la définition de l'« évolution », la seconde l'identification de la catégorie « espèce » pertinente en économie, la troisième le mode opératoire au travail dans le processus de "sélection naturelle ", c'est-à-dire celui des «variations » des « caractères » et de leur processus de "sélection », le tout constituant une «dérivation » (variation-sélection). La dernière question a trait au processus de «transmission » des nouveaux caractères sélectionnés, perpétuant ainsi l'espèce transformée. Les réponses de Veblen à ces quatre questions expliquent l'architecture générale de sa généalogie des institutions économiques.

Thorstein Veblen offers an approach of the evolution of the economic institutions based on the methodological darwinism. Veblen then has to answer four important methodological questions. The first one concerns the definition of the "evolution", second the identification of the category relevant "species" in economy, the third the modus operandi in the work in the process of "natural selection", that is that of the "variations" of the "characters" and their process of "selection", it quite constituting a "diversion" (variation-selection). The last question concerns the process of "transmission" of the new selected characters, so immortalizing the transformed species. The answers of Veblen to these four questions explain the general architecture of its genealogy of the economic institutions. 
INDEX

Mots-clés : Veblen (Thorstein), darwinisme méthodologique, institution économique, évolution, variation, sélection, transmission

Keywords : Veblen (Thorstein), methodological darwinism, economic institutions, evolution, variation, selection, transmission

Code JEL B15 - Historical • Institutional • Evolutionary, B31 - Individuals, B52 - Institutional • Evolutionary

\section{AUTEUR}

JEAN-JACQUES GISLAIN

CRISES - Québec, PHARE - Paris 1 